

\title{
Stochastic finite element: a non intrusive approach by regression
}

\author{
Marc Berveiller, Bruno Sudret, Maurice Lemaire
}

\section{To cite this version:}

Marc Berveiller, Bruno Sudret, Maurice Lemaire. Stochastic finite element: a non intrusive approach by regression. Revue Européenne de Mécanique Numérique/European Journal of Computational Mechanics, 2006, 15 (1-2-3), pp.81-92. 10.3166/remn.15.81-92 . hal-01665506

\section{HAL Id: hal-01665506 https://hal.science/hal-01665506}

Submitted on 16 Dec 2017

HAL is a multi-disciplinary open access archive for the deposit and dissemination of scientific research documents, whether they are published or not. The documents may come from teaching and research institutions in France or abroad, or from public or private research centers.
L'archive ouverte pluridisciplinaire HAL, est destinée au dépôt et à la diffusion de documents scientifiques de niveau recherche, publiés ou non, émanant des établissements d'enseignement et de recherche français ou étrangers, des laboratoires publics ou privés. 


\title{
Stochastic finite element : a non intrusive approach by regression
}

\author{
Marc Berveiller* - Bruno Sudret ${ }^{*}$ - Maurice Lemaire** \\ *Electricité de France - R\&D Division, Site des Renardières, \\ F-77818 Moret-sur-Loing, \\ ${ }^{* *}$ LaMI - UBP \& IFMA \\ Institut Français de Mécanique Avancée, \\ Campus de Clermont-Ferrand - Les Cézeaux, BP265, \\ F-63175 Aubière \\ \{marc.berveiller, bruno.sudret\}@edf.fr \\ maurice.lemaire@ifma.fr
}

ABSTRACT. The stochastic finite element method allows to solve stochastic boundary value problems where material properties and loads are random. The method is based on the expansion of the mechanical response onto the so-called polynomial chaos. In this paper, a non intrusive method based on a least-squares minimization procedure is presented. This method is illustrated by the study of the settlement of a foundation. Different analysis are proposed: the computation of the statistical moments of the response, a reliability analysis and a parametric sensitivity analysis.

RÉSUMÉ. La méthode des éléments finis stochastiques permet de résoudre des problèmes aux limites dans lesquels les propriétés des matériaux et le chargement sont aléatoires. Cette méthode est basée sur le développement de la réponse sur la base du chaos polynomial. Dans ce papier, une méthode "non intrusive" basée sur une minimisation au sens des moindres carrés est présentée. Cette méthode est appliquée à l'étude du tassement d'une fondation. On montre comment obtenir les moments statistiques de la réponse et comment effectuer une analyse de fiabilité.

KEYWORDS: stochastic finite element, non intrusive method, polynomial chaos, stochastic response surface method

MOTS-CLÉS : Eléments finis stochastiques, méthode non intrusive, chaos polynomial, surfaces de réponse stochastiques 


\section{Introduction}

The Stochastic Finite Element Method (SFEM) developed by (Ghanem et al., 1991) allows to solve stochastic boundary value problems involving spatially randomly varying material properties usually described as Gaussian or lognormal random fields. The method is based on the discretization of the input random fields and the expansion of the mechanical response onto the so-called polynomial chaos. A similar procedure allowing to model random material properties and loads by means of an arbitrary number of random variables of any type has been recently proposed (Sudret et al., 2004, Sudret et al., 2005, Berveiller, 2005). In both cases, the coefficients of the response expansion are computed using a Galerkin procedure, which leads to a linear system whose size is equal to the number of degrees of freedom of the finite element model multiplied by the number of coefficients retained in the response expansion. The output of this method is the expansion of response quantities $S(\theta)$ (such as displacement, strain or stress components) onto the polynomial chaos.

$$
S(\theta)=\sum_{j=0}^{P-1} s_{j} \Psi_{j}\left(\left\{\xi_{k}(\theta)\right\}_{k=1}^{M}\right)
$$

where $s_{j}$ are the coefficients to be determined, $\left\{\Psi_{0}, \cdots, \Psi_{P-1}\right\}$ are multidimensionnal Hermite polynomials of order less or equal than $p,\left\{\xi_{k}(\theta)\right\}_{k=1}^{M}$ are standard Gaussian random variables, $M$ is the number of random input parameters and $P$ denotes the size of the polynomial chaos basis, which is given by:

$$
P=\frac{(M+p) !}{M ! p !}
$$

In this paper, a non intrusive method (called regression method) is proposed for computing these coefficients using a series of deterministic analysis.

\section{Non intrusive regression method}

The non intrusive regression method presented in this section is based on a leastsquares minimization between the exact solution and the approximate solution based on the polynomial chaos (Isukapalli, 1999, Mahadevan et al., 2003, Berveiller et al., 2004, Berveiller, 2005). First the input random variables (gathered in a random vector $\underline{X}$ whose joint PDF (Probability Density Function) is prescribed) are transformed into a standard Gaussian vector $\underline{\xi}$. If these $M$ variables are independent, the one-to-one mapping reads :

$$
\xi_{i}=\Phi^{-1}\left(F_{i}\left(X_{i}\right)\right)
$$

where $\Phi$ is the standard Gaussian CDF (Cumulative Density Function) and $\left\{F_{i}\left(X_{i}\right), i=1, \cdots, M\right\}$ are the marginal CDF of the $X_{i}$ 's. Let us denote by $\left\{\underline{\xi}^{(k)}, k=1, \cdots, n\right\} n$ outcomes of the standard Gaussian random vector $\underline{\xi}$. For each 
outcome $\underline{\xi}^{(k)}$, the isoprobabilistic transform yields a vector of input random variables $\underline{X}^{(k)}$ (Eq.(3)). Using a classical finite element code, the response vector $\underline{S}^{(k)}$ can be computed. According to Eq.(1), each scalar component of the response $S$ (displacement, strain, stress, etc.) may be approximated as follows:

$$
S \approx \tilde{S}(\underline{\xi})=\sum_{j=0}^{P-1} s_{j} \Psi_{j}(\underline{\xi})
$$

where $\left\{s_{j}\right\}_{j=0}^{P-1}$ are coefficients to be computed. The regression method consists in finding the set of coefficients that minimizes the difference:

$$
\Delta S=\sum_{k=1}^{n}\left[S^{(k)}-\tilde{S}\left(\underline{\xi}^{(k)}\right)\right]^{2}
$$

These coefficients are solution of the following linear system:

$$
\begin{gathered}
\left(\begin{array}{ccc}
\sum_{k=1}^{n} \Psi_{0}\left(\underline{\xi}^{(k)}\right) \Psi_{0}\left(\underline{\xi}^{(k)}\right) & \ldots & \sum_{k=1}^{n} \Psi_{0}\left(\underline{\xi}^{(k)}\right) \Psi_{P-1}\left(\underline{\xi}^{(k)}\right) \\
\vdots & \ddots & \vdots \\
\sum_{k=1}^{n} \Psi_{P-1}\left(\underline{\xi}^{(k)}\right) \Psi_{0}\left(\underline{\xi}^{(k)}\right) & \cdots & \sum_{k=1}^{n} \Psi_{P-1}\left(\underline{\xi}^{(k)}\right) \Psi_{P-1}\left(\underline{\xi}^{(k)}\right)
\end{array}\right) \cdot\left(\begin{array}{c}
s_{0} \\
\vdots \\
s_{P-1}
\end{array}\right) \\
=\left(\begin{array}{c}
\sum_{k=1}^{n} S^{(k)} \Psi_{0}\left(\underline{\xi}^{(k)}\right) \\
\vdots \\
\sum_{k=1}^{n} S^{(k)} \Psi_{P-1}\left(\underline{\xi}^{(k)}\right)
\end{array}\right)
\end{gathered}
$$

Note that the $P \times P$ matrix on the left hand size is evaluated once and for all. Moreover it is independent on the mechanical problem under consideration. Then the coefficients of the expansion of each response quantity $s^{i}$ are obtained by the resolution of the linear system Eq.(6).

The crucial point in this approach is to properly select the collocation points, i.e. the outcomes $\left\{\xi^{(k)}, k=1, \cdots, n\right\}$. Note that $n \geq P$ is required so that a solution of (6) exist. (Webster et al., 1996) and (Isukapalli, 1999) choose for each input variable the $(p+1)$ roots of the $(p+1)$-th order Hermite polynomial $H_{p+1}(x)$, and then built $(p+1)^{M}$ vectors of length $M$ using all possible combinations between theses different roots (Figure 1). Then they select $n$ outcomes $\left\{\underline{\xi}^{(k)}, k=1, \cdots, n\right\}$ out of these $(p+1)^{M}$ possible combinations as follows : 
- (Webster et al., 1996) selected $n=P+1$ and the $(P+1)$ outcomes which $\operatorname{maximize} \varphi_{n}\left(\underline{\xi}^{(k)}\right)=(2 \pi)^{-M / 2} \exp \left[-1 / 2\left\|\underline{\xi}^{(k)}\right\|^{2}\right]$. This corresponds to selecting the points that minimize $\|\underline{\xi}\|^{2}$.

- (Isukapalli, 1999) selected by the same method $2(P+1)$ outcomes, adding the null vector $\underline{\xi}=\underline{0}$ if it is not already included in the experimental design.

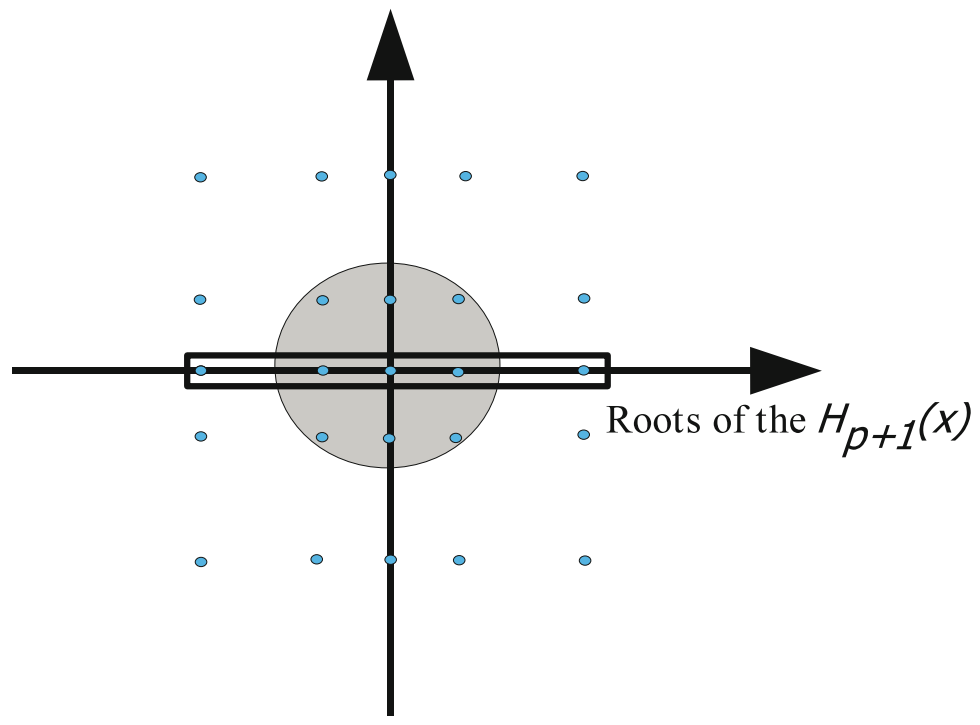

Figure 1. Scheme of the selection of the collocation points ( $M=2, p=4, n=9)$

No indication of the reasons of these choices for $n$ could be found in the literature. In the sequel, a parametric study is carried out to investigate the influence of $n$ on the accuracy of the results. Indeed, using the selection method mentioned above, it is easy to increase the accuracy by taking more collocation points, without losing the benefit of previous computations. It is also possible to define an error estimator for any response quantity $S$. Suppose that we have $n=k P$ collocation points, where $k=1,2, \cdots$, and that the minimal $k$ for a given accuracy is looked after. For each $k \geq 1$, an estimation of the response quantity $S$ (assumed not vanishing) is computed together with a convergence rate $\varepsilon_{k}$ :

$$
\varepsilon_{k}=\left|\frac{S_{k+1}-S_{k}}{S_{k}}\right|
$$

If this rate is smaller than a tolerance, say $1 \%$, the regression scheme is said to be accurate. Otherwise $P$ new collocation points are added to the scheme (leading to $P$ additional finite element runs). The procedure is followed up until convergence. 


\section{Application example : settlement of a foundation}

\subsection{Description}

Let us consider an elastic soil mass made of two layers of different isotropic linear elastic materials lying on a rigid substratum (Figure 2(a)). A foundation on this soil mass is modeled as two uniform pressure loads $P_{1}$ and $P_{2}$ applied respectively over a length $2 B_{1}$ and $2 B_{2}$ of the free surface (Figure $2(b)$ ). Due to the symmetry, half of the structure is modeled by finite element. The mesh comprises 99 nodes and 804 -node elements which allows a 1\%-accurate evaluation of the maximal settlement compared to a reference solution.



(a)



(b)

Figure 2. (a) : Scheme of the foundation - (b) : Mesh of the foundation

In the sequel, three different analysEs of this problem are performed. First, the statistical moments of the maximum settlement are computed. Then a reliability analysis with respect to admissible settlements is carried out. Finally, the approach is used in a purely deterministic context, where the parameters are given interval values.

\subsection{Statistical moments of the response}

In this section, the six parameters (namely Young modulus and Poisson ration in each layer and two loads) are modelled as random variables described in Table1. We consider a polynomial chaos of order three. Thus $P=\frac{(6+3) !}{6 ! 3 !}=84$ coefficients are to be determined using the non intrusive method. We are interested in the evaluation of the maximum settlement of the foundation obtained at point $\mathrm{A}$ and denoted by $u_{A}$.

The four statistical moments of the maximal settlement of the foundation obtained by the non intrusive method are compared to reference results. From Eq.(1), the statistical moments of any response quantity can be computed analytically. The mean and the variance of $S$ are given by:

$$
\mathrm{E}[S]=s_{0}
$$


Table 1. Definition of the input random variables for moments and reliability analysis

\begin{tabular}{lcccc}
\hline Parameter & Notation & Distribution & Mean & Coeff. Var. \\
\hline Young modulus upper layer & $E_{1}$ & Lognormal & $50 \mathrm{MPa}$ & $20 \%$ \\
Young modulus lower layer & $E_{2}$ & Lognormal & $70 \mathrm{MPa}$ & $20 \%$ \\
Poisson ratio upper layer & $\nu_{1}$ & Uniform $[0.28 ; 0.32]$ & 0.3 & $4 \%$ \\
Poisson ratio lower layer & $\nu_{2}$ & Uniform [0.28;0.32] & 0.3 & $4 \%$ \\
Load \#1 & $P_{1}$ & Weibull & $0.2 \mathrm{MPa}$ & $30 \%$ \\
Load \#2 & $P_{2}$ & Lognormal & $0.4 \mathrm{MPa}$ & $20 \%$ \\
\hline
\end{tabular}

$$
\operatorname{Var}[S]=\sigma_{S}^{2}=\sum_{i=1}^{P-1} \mathrm{E}\left[\Psi_{i}^{2}\right] s_{i}^{2}
$$

The skewness and the kurtosis coefficients are given by:

$$
\begin{aligned}
& \delta_{S}=\frac{1}{\sigma_{S}^{3}} \sum_{i=1}^{P-1} \sum_{j=1}^{P-1} \sum_{k=1}^{P-1} \mathrm{E}\left[\Psi_{i} \Psi_{j} \Psi_{k}\right] s_{i} s_{j} s_{k} \\
& \kappa_{S}=\frac{1}{\sigma_{S}^{4}} \sum_{i=1}^{P-1} \sum_{j=1}^{P-1} \sum_{k=1}^{P-1} \sum_{l=1}^{P-1} \mathrm{E}\left[\Psi_{i} \Psi_{j} \Psi_{k} \Psi_{l}\right] s_{i} s_{j} s_{k} s_{l}
\end{aligned}
$$

The analytical formulae to compute the expectation of products of $\Psi_{j}$ polynomials are given in (Berveiller, 2005). Reference results are obtained by Monte Carlo simulation (10000 samples) using a direct coupling between the finite element code Code_Aster ${ }^{1}{ }^{1}$ and the probabilistic code PROBAN. Figure 3 shows the evolution of the statistical moments versus the number of samples in the Monte Carlo simulation. Table 2 gathers the reference results and those obtained by the non intrusive method. With the non intrusive regression method, good results are obtained for the four first moments if more than 420 points (i.e. $(M-1) P$ points) are taken. Figure 4 presents the evolution of the four first statistical moments (divided by the converged value in the regression method) versus the number of collocation points. We can note that from 420 points (i.e. $5 \times 84 \equiv(M-1) P$ points), results have converged. Note that the skewness and kurtosis coefficients obtained by Monte Carlo simulation slightly differ from the regression results.

\subsection{Reliability analysis}

In reliability analysis, the failure criterion of a structure is defined in terms of a limit state function $g(\underline{X}, S(\underline{X}))$ which may depend both on basic random variables $\underline{X}$

1. http://www.code-aster.org 
Table 2. Statistical moments of the maximal settlement

\begin{tabular}{lcccccc}
\hline & MC Simulation & \multicolumn{5}{c}{ Non intrusive method } \\
\#FE runs & 10000 & 84 & 168 & 336 & 420 & 4096 \\
\hline Mean & -1.097 & -0.566 & -0.735 & -1.040 & -1.108 & -1.108 \\
Std. Dev. & 0.0256 & 0.0802 & 0.0747 & 0.0439 & 0.0250 & 0.0250 \\
Skewness & -0.6019 & 1.6664 & 1.9638 & 2.3967 & -0.5182 & -0.5303 \\
Kurtosis & 3.7214 & 8.7149 & 10.9272 & 23.7676 & 3.4826 & 3.5095 \\
\hline
\end{tabular}

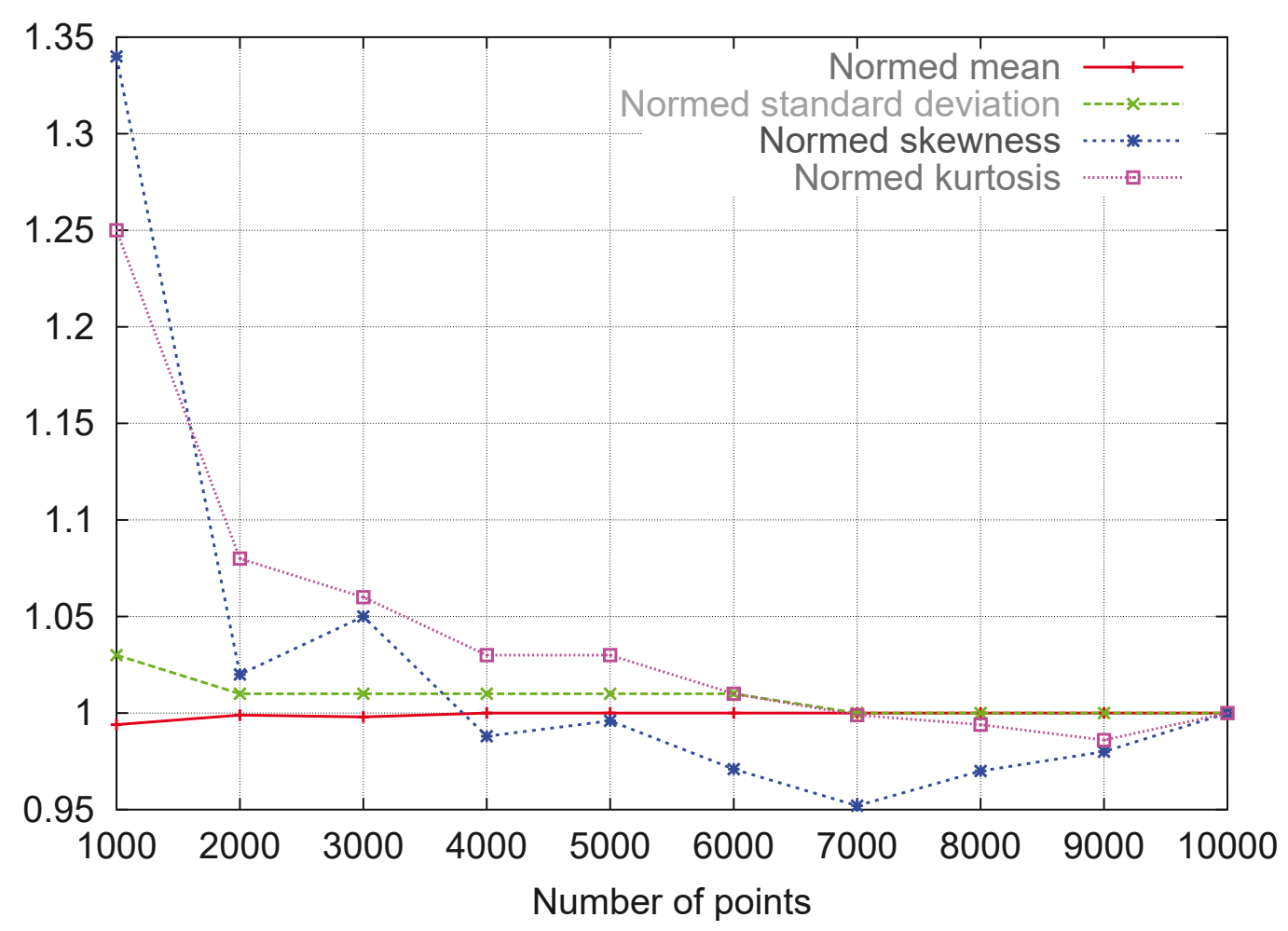

Figure 3. Evolution of moments obtained by direct coupling vs. the number of samples in the Monte Carlo simulation

and response quantities $S(\underline{X})$. The domain defined by $g(\underline{X}, S(\underline{X}))<0$ is the failure domain and $g(\underline{X}, S(\underline{X}))>0$ defines the safe domain. The boundary between these two domains is the limit state surface. When performing a stochastic finite element analysis, the true limit state function is replaced by its polynomial approximation onto the polynomial chaos. Thus the approximation is defined by definition in the so-called standard normal space, which is the space used for the First Order Reliability Method (FORM) in reliability analysis:

$$
g(\underline{X}, S(\underline{X})) \equiv \tilde{g}\left(\left\{\xi_{k}\right\}_{k=1}^{M}, \sum_{j=0}^{P-1} \underline{S}_{j} \Psi_{j}\left(\left\{\xi_{k}\right\}_{k=1}^{M}\right)\right)
$$




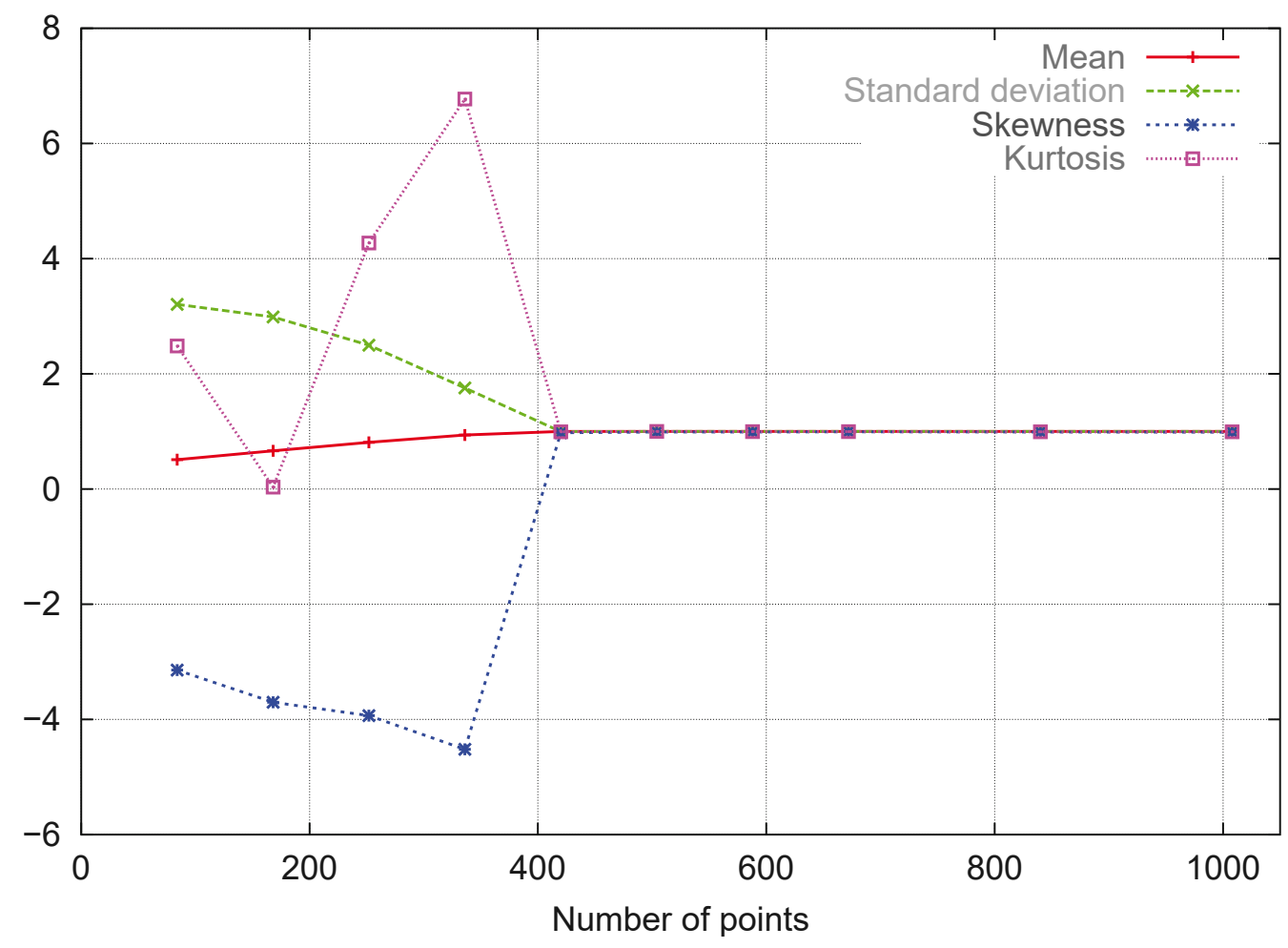

Figure 4. Evolution of moments vs. the number of collocation points in the non intrusive regression method

Thus the reliability problem, which is already formulated in the standard Gaussian space, may be solved by any available method including Monte-Carlo Simulation, FORM/SORM, Importance Sampling (IS), etc. (Ditlevsen et al., 1996).

The reliability of the foundation with respect to the maximum admissible settlement $\bar{u}$ is investigated. Hence the limit state function can be written:

$$
g(\underline{X})=\bar{u}-u_{A}\left(E_{1}, E_{2}, \nu_{1}, \nu_{2}, P_{1}, P_{2}\right)
$$

where $u_{A}\left(E_{1}, E_{2}, \nu_{1}, \nu_{2}, P_{1}, P_{2}\right)$ is the maximal displacement. The failure probability is computed by FORM and importance sampling for different values of $\bar{u}$ using the two strategies mentioned above, namely:

- a direct coupling between the finite element code Code_Aster $($ and the probabilistic code PROBAN by using FORM and importance sampling. Eq.(13) is used for this purpose, when $u_{A}(\cdot)$ is obtained with the finite element code. 1,000 samples are used in IS allowing a coefficient of variation of the simulation of $1 \%$.

- the non intrusive regression method followed by a FORM reliability analysis and importance sampling (1,000 samples and a coefficient of variation of $1 \%$ for the simulation). Note that FORM as well as IS are performed using analytical limite state function in this case :

$$
\tilde{g}(\underline{\xi})=\bar{u}-\sum_{j=0}^{83} u_{A j} \Psi_{j}\left(\left\{\xi_{k}\right\}_{k=1}^{6}\right)
$$


In this approach, the impact of the number of collocation points used to compute the $u_{A j}$ 's onto the accuracy is studied.

Table 3 presents the probability of failure obtained by direct coupling and by regression using various numbers of collocation points. Figure 5 shows the evolution of the ratio between the logarithm of the probability of failure (divided by the logarithm of the converged probability of failure) versus the number of collocation points for several values of the maximum admissible settlement $\bar{u}$. Again, accurate results are obtained when using 420 collocation points or more for different values of the failure probability (from $10^{-1}$ to $10^{-4}$ ). When taking less than 420 points, results are inaccurate. When taking more than 420 points, the accuracy is not improved. It seems that the best compromise between accuracy and efficiency is $(M-1) P$ collocation points in this case, as it was also observed in the previous section.

Table 3. Failure probability $P_{f}$

\begin{tabular}{lcccccc}
\hline $\bar{u}$ & Direct & \multicolumn{5}{c}{ Non intrusive } \\
$(\mathrm{cm})$ & Coupling & 84 & 168 & 336 & 420 & 4096 \\
\hline 12 & $3.09 .10^{-1}$ & $1.62 .10^{-1}$ & $2.71 .10^{-1}$ & $3.31 .10^{-1}$ & $3.23 .10^{-1}$ & $3.32 .10^{-1}$ \\
15 & $6.83 .10^{-2}$ & $6.77 .10^{-2}$ & $6.90 .10^{-2}$ & $8.43 .10^{-2}$ & $6.73 .10^{-2}$ & $6.93 .10^{-2}$ \\
20 & $2.13 .10^{-3}$ & - & $9.95 .10^{-5}$ & $8.22 .10^{-4}$ & $2.01 .10^{-3}$ & $1.98 .10^{-3}$ \\
22 & $4.61 .10^{-4}$ & - & $7.47 .10^{-7}$ & $1.31 .10^{-4}$ & $3.80 .10^{-4}$ & $4.24 .10^{-4}$ \\
\hline
\end{tabular}

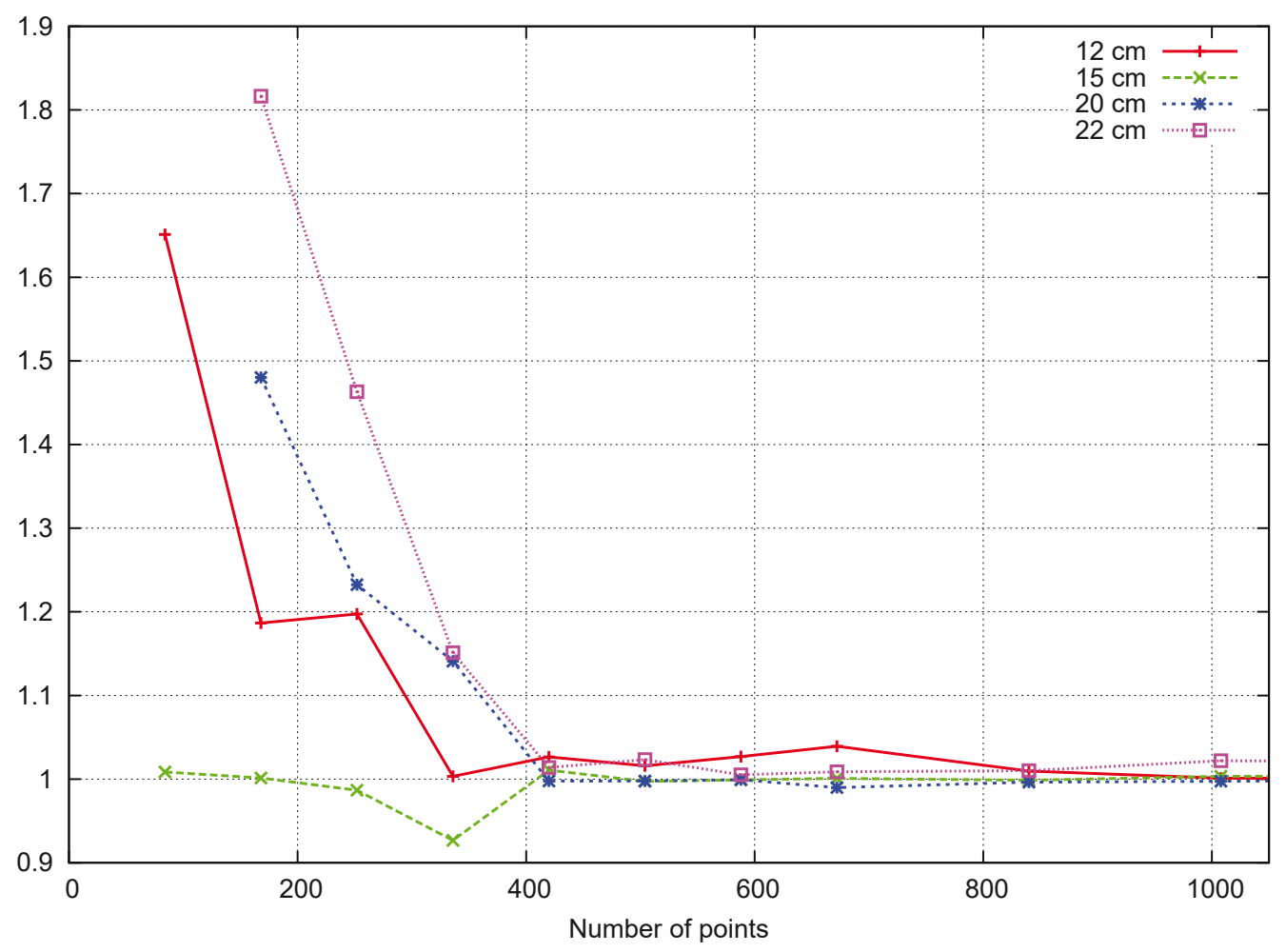

Figure 5. Evolution of the logarithm of the failure probability divided by the converged value versus the number of collocation points 


\subsection{Deterministic parametric analysis}

In this section, the formalism of stochastic finite element is used to build a deterministic response surface of the 6-parameter model. In this sense, the input parameters are given a range of variation $[a ; b]$. To be able to apply the proposed method, these intervals are then considered as support of uniform random variables (Table 4).

Table 4. Definition of input parameters for the parametric analysis

\begin{tabular}{lcc}
\hline Parameter & Notation & Interval \\
\hline Young's modulus upper layer & $E_{1}$ & {$[50 ; 70] \mathrm{MPa}$} \\
Young's modulus lower layer & $E_{2}$ & {$[70 ; 90] \mathrm{MPa}$} \\
Poisson's ratio upper layer & $\nu_{1}$ & {$[0.1 ; 0.4]$} \\
Poisson's ratio lower layer & $\nu_{2}$ & {$[0.1 ; 0.4]$} \\
Load \#1 & $P_{1}$ & {$[0.1 ; 0.3] \mathrm{MPa}$} \\
Load \#2 & $P_{2}$ & {$[0.1 ; 0.3] \mathrm{MPa}$} \\
\hline
\end{tabular}

Figure 6(a) shows the relative error between a deterministic finite element analysis and the approximation of the settlement $u_{A}$ by a third order polynomial chaos using 420 collocation points. We can note that the maximum error is about 4\%. Figure 6(b) shows the Henry's line (Saporta, 1990) of the residual which allows to determine whether the residual follows a Gaussian distribution. The straight line (dotted) draw on this figure is the fitted line to the Henry's line. We can also determine the mean and the standard deviation of the residual fitted to a Gaussian distribution and compared them with those obtained empirically. The mean of the residual is equal to $-3.96 .10^{-6} \mathrm{~m}$ (the mean settlement is $-0.082 \mathrm{~m}$ ). The standard deviation is equal to $7.94 .10^{-4}$. The skewness coefficient and the kurtosis coefficient are respectively equal to -0.56 and 0.64. For a Gaussian distribution these values are respectively 0 and 3. Values of skewness and kurtosis coefficients allow to conclude that the residual is not fitted to a Gaussian distribution. The Table 5 gathers the $R^{2}$ coefficient (Saporta, 1990) of the regression versus the number of collocation points. Again this coefficient shows an excellent quality of the regression whatever the number of points. It is concluded that $P$ collocation points are sufficient to build a deterministic response surface of the foundation. Note that the deterministic response surface built using the polynomial chaos is now straight forward to use. For any values of the input parameters (in the range given in Table 4), the corresponding standard normal values are computed using Eq.(3). Then the quantities are used to evaluate the polynomial expansion.

Table 5. $R^{2}$ coefficient

\begin{tabular}{cccccc}
\hline Number of collocation points & 84 & 168 & 252 & 336 & 420 \\
$R^{2}$ & 0.999 & 0.992 & 0.993 & 0.993 & 0.993 \\
\hline
\end{tabular}




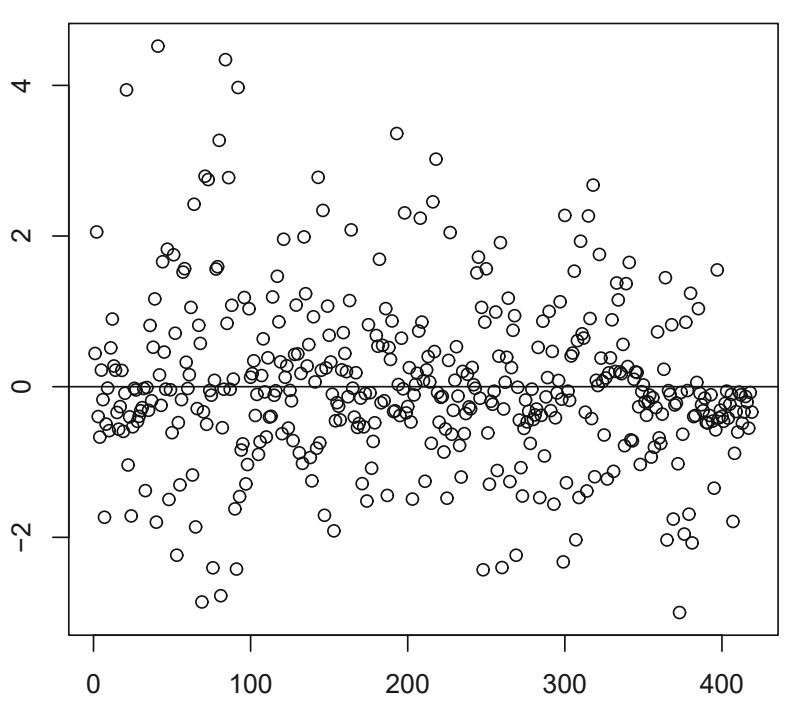

(a)

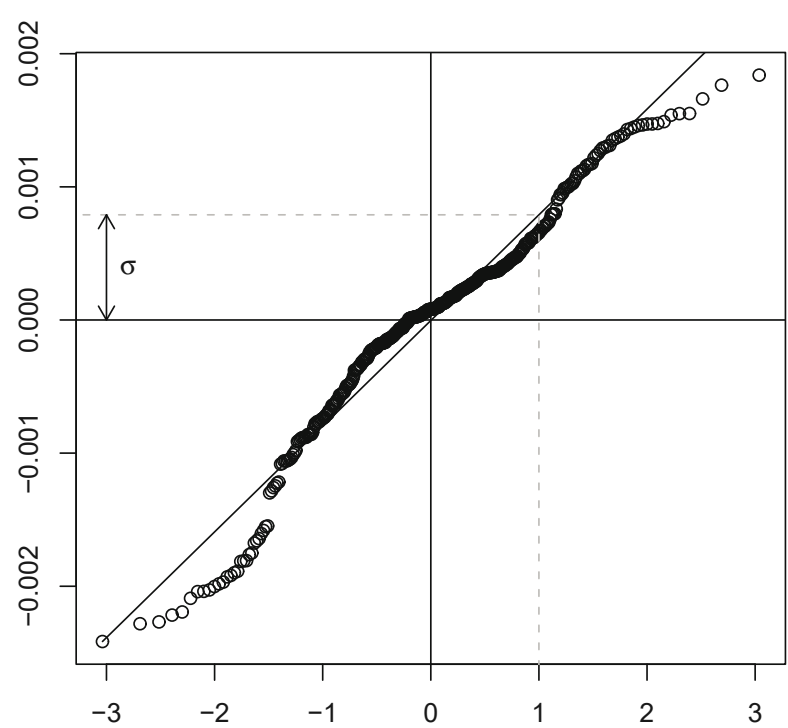

(b)

Figure 6. (a) : Relative error (\%) - (b) : Henry's line (420 collocation points)

\section{Conclusion}

The paper presents a non intrusive method to compute coefficients of the polynomial series expansion of the response in stochastic finite element analysis. This method is based on a least-squares minimization of the distance between the exact solution and the polynomial expansion. The result is interpreted as a stochastic polynomial response surface. The collocation points are selected among $M$-uplets of roots of Hermite polynomials. The method is illustrated by the analysis of the settlement of a foundation. Various analysis are performed such as computation of the statistical moments of the response and reliability analysis. Based on the selection scheme, it appears that a number of points equal to $(M-1)$ times the size of the polynomial chaos (i.e. 420 points in the application example) provides excellent accuracy both in the mean region (moments of the response) and in the tail (probability of exceedance of a threshold). As far as a deterministic parametric study is concerned, $P$ collocation points are sufficient to get an accurate deterministic response surface. The great advantage of the non intrusive approach compared to the classical Galerkin approach is that only deterministic finite element models are used. Hence the full non linear capabilities of the code may be used without additional implementation (see for instance (Berveiller et al., 2005)). Note that if several output quantities are of interest, the marginal cost to estimate the response coefficients is low. Indeed the deterministic finite element analysis are usually the expensive part of the evaluation. 


\section{References}

Berveiller M., Eléments finis stochastiques : approches intrusive et non intrusive pour des analyses de fiabilité, PhD thesis, Université Blaise Pascal - Clermont Ferrand, 2005.

Berveiller M., Sudret B., Lemaire M., « Presentation of two methods for computing the response coefficients in stochastic finite element analysis », Proc. 9th ASCE specialty Conference on Probabilistic Mechanics and Structural Reliability, Albuquerque, USA, 2004.

Berveiller M., Sudret B., Lemaire M., « Non linear non intrusive stochastic finite element method - Application to a fracture mechanics problem », Proc. 9th Int. Conf. Struct. Safety and Reliability (ICOSSAR'2005), Rome, Italie, 2005.

Ditlevsen O., Madsen H., Structural reliability methods, J. Wiley and Sons, Chichester, 1996.

Ghanem R.-G., Spanos P.-D., Stochastic finite elements - A spectral approach, Springer Verlag, 1991.

Isukapalli S. S., Uncertainty Analysis of Transport-Transformation Models, PhD thesis, The State University of New Jersey, 1999.

Mahadevan S., Huang S., Rebba R., « A stochastic response surface method for random field problems », Proc. ICASP9 "Applications of Statistics and Probability to Civil Engineering Reliability and Risk Analysis", vol. 1, p. 177-184, 2003.

Saporta G., Probabilités, analyse des données et statistique, Editions Technip, 1990.

Sudret B., Berveiller M., Lemaire M., « Eléments finis stochastiques en élasticité linéaire », C. R. Mécanique, vol. 332, p. 531-537, 2004.

Sudret B., Berveiller M., Lemaire M., « A stochastic finite element procedure for moment and reliability analysis », Rev. Eur. El. Finis, 2005. soumis pour publication.

Webster M., Tatang M., McRae G., Application of the Probabilistic Collocation Method for an Uncertainty Analysis of a Simple Ocean Model, Technical report, MIT Joint Program on the Science and Policy of Global Change Reports Series No. 4, Massachusetts Institute of Technology, 1996. 